\title{
A theoretical and empirical model based RSSI localization algorithm
}

\author{
Pingfang $\mathrm{Hu}^{1,}$, Suyu Huang ${ }^{2, \mathrm{~b}}$ \\ ${ }^{1}$ School of computer, Wuhan Donghu University, Wuhan 430212, China \\ ${ }^{2}$ School of computer, Wuhan Donghu University, Wuhan 430212, China \\ a357793685@qq.com, b604656446 @qq.com,
}

\begin{abstract}
Keywords: wireless sensor networks, localization algorithm, continuous positioning,
\end{abstract} one-dimensional.

\begin{abstract}
Aiming at the application fields of road monitoring, pipeline transmission and line transmission, a method of one-dimensional continuous positioning based on RSSI value is proposed by combining theoretical model and empirical model. Compared with RADAR and 1-DEMRSSI method, this method improves the positioning accuracy under the condition that the computational processing workload increases slightly. At the same time, the method can greatly reduce the number of reference test points.
\end{abstract}

\section{Introduction}

Node localization is a very important application support technology in sensor networks[1]. Sensor network node localization has become a very important research direction and hot issues. Node localization algorithms in wireless sensor networks with different angles can have a variety of classification methods[2], such as absolute and relative positioning, centralized computing and distributed computing, ranging and positioning based on range free. In localization based on received signal strength indication algorithm, signal strength is known of the transmitting node, the receiving node according to the received signal strength, calculates the propagation loss of a signal, the transmission loss into the distance using the model to calculate the position of the node using the existing algorithm. RADAR[3] is a classic indoor positioning system based on RSSI technology to determine the location of user nodes in the floor. The system has deployed three base stations in the monitoring area, the user node periodically transmit a beacon signal, and the signal strength is known, the base station calculates the propagation loss according to the received signal strength, calculate the transmission distance is obtained after the user location. Based on the RADAR positioning algorithm, improved 1-DEMRSSI algorithm for special application scenarios to achieve one-dimensional positioning[4]. Based on the idea of one-dimensional localization, the algorithm sets multiple reference test points between two base stations, and establishes the signal strength ratio records of the two base stations at each test point. The simulation results show that it has good flexibility and practicability in one-dimensional application field.

The 1-D EMRSSI algorithm does not consider the continuous positioning problem between reference test points, based on the 1-D EMRSSI algorithm, puts forward 1-DEMMRSSI (1-Dimension Empirical and Mathematical Model RSSI Localization) algorithm, this algorithm firstly according to the empirical model to determine the unknown nodes in between which two test points. Then the continuous positioning calculation between the two test points by using the theoretical model, obtain the location information of an unknown node.

\section{One-dimensional RSSI localization algorithm combining theoretical and empirical models}

Sensor network localization algorithm is generally based on two-dimensional, and in the road monitoring, pipeline transmission, line transmission and other applications, monitoring objects tend to be linear distribution, so the need for one-dimensional positioning. Two base stations are arranged in the monitoring area, and the base station and the user are all wireless sensor network nodes. The two base stations periodically transmit positioning singals, and the user receives the continuous 
positioning according to the ratio of the detected signal intensity. system structure shown in figure 1 . Node wireless signal attenuation using logarithmic distance decay model, as shown in the following.

$$
P(d)[d B m]=P\left(d_{0}\right)[d B m]-10^{\alpha} \log \left(\frac{d}{d_{0}}\right)
$$

In the formula, $\mathrm{d}$ is the user of a base station distance; $\alpha$ is path loss rate and distance with growth; $P\left(d_{0}\right)$ said the signal power transmitting station at a reference distance on the $d 0 ; P(d)$ is the distance for d signal power attenuation.

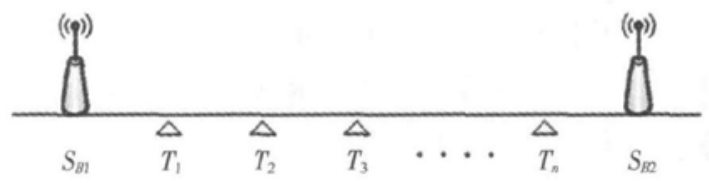

图 1 系统结构示意图

Fig. 1 Architecture of the system

As shown in Figure 1, even between two base stations or random set several test points $T_{1}$, $T_{2}, \ldots T_{n}$., between the two base station distance is $D$, a reference point $T_{i}(i=1, \ldots, n)$ to the two base station distance were $D_{i 1}$ and $D_{i 2}$, received two a base station transmits the wireless signal strength of $\mathrm{S}_{\mathrm{i} 1}$ and $\mathrm{S}_{\mathrm{i} 2}$, according to the formula (1) is:

$$
\left\{\begin{array}{l}
S_{i 1}=P\left(d_{0}\right)-10^{\alpha} \log \left(\frac{D_{i 1}}{D}\right) \\
S_{i 2}=P\left(d_{0}\right)-10^{\alpha} \log \left(\frac{D 2}{D}\right)
\end{array}\right.
$$

Therefore, the signal intensity radio of the two base stations received by the reference point $T_{i}$ is:

$$
R_{i}=\frac{S_{i 1}}{S_{i 2}}
$$

When positioning, the user received two base station signal strength were $S_{\mathrm{u} 1}$ and $S_{\mathrm{u} 2}$, the ratio of $\mathrm{R}_{\mathrm{u}}$ is defined as follows:

$$
R_{u}=\frac{S_{u 1}}{S_{u 2}}
$$

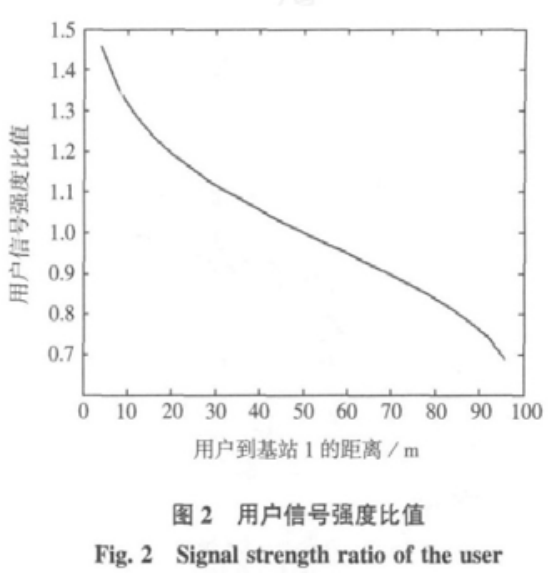

According to the wireless signal attenuation model, can draw the user signal strength ratio, with the user position changes, as shown in figure 2. Can be seen from the figure, With the increase in the distance between the user and the base station 1, the ratio of user signal strength continues Reduced, monotonic variation. Therefore, the user signal ratio can be obtained from the measurement, determine which two adjacent test points the user is located between.

After determining which user is located between the two test points, you can Continuous positioning. Similarly, as shown in Figure 2, the ratio of user signal strength Values vary monotonically with user location. Because the positioning area is set A reference test point, the distance of adjacent test points is relatively small, in order to reduce the user Locate the amount of 
computation to reduce energy consumption between each adjacent test point An approximate meter with a linear variation of signal strength ratio and user position Calculation method. User location calculations as shown in formula (5), Xu for user computing Location, $\mathrm{X}_{\mathrm{i}-1}$ for $\mathrm{i}-1$ reference test point locations, $R_{i-1}, R_{i}$, respectively, for the Senate Test point $i-1$ and $i$ base station signal strength ratio, $\mathrm{Ru}$ for the user to obtain the base station signal strength ratio, $\mathrm{d}_{\mathrm{i}-1, \mathrm{i}}$ for the distance between the two reference test points $\mathrm{i}-1$ and $\mathrm{i}$.

$$
X_{u}=X_{i-1}+\frac{R_{u}-R_{i-1}}{R_{i-1}-R_{i}} \times d_{i-1, i}
$$

The calculation method of using approximate theory, the positioning errors are shown in Table 1, table positioning area is $0 \sim 100 \mathrm{~m}$, the test points are evenly distributed, divided by the test will locate the area of the positioning section number is $n+1$, positioning error and positioning the number of segments is directly related to. When the number of positioning segments increases, the theoretical positioning error of linear approximation is reduced.

Table 1 Theoretical localization error of the linear approximate method

\begin{tabular}{c|c|c|c|c|c|c}
\hline positioning segments & 5 & 10 & 20 & 30 & 40 & 50 \\
\hline $\begin{array}{c}\text { Theoretical positioning error } \\
\text { Maximum(m) }\end{array}$ & 1.18 & 0.74 & 0.40 & 0.29 & 0.23 & 0.18 \\
\hline
\end{tabular}

Low power consumption is one of the main goals of wireless sensor network design. The energy consumption closely related to sensor network localization can be divided into computing, communication, storage and other overhead. In the three positioning methods, the communication energy overhead is basically the same. Because of different positioning algorithms, the energy consumption of computation processing is different. Assuming there are $\mathrm{n}$ test points, RADAR method positioning once need $n+3$ addition and subtraction method, the 3 multiplication and $N$ comparison. 1 - DEMRSSI method positioning requires 1 times division, $\mathrm{n}$ subtraction, $\mathrm{n}$ comparison. A positioning of this method requires $n+3$ addition and subtraction, multiplication and division, 4 times n. From the computational amount of the calculation method and the RADAR method is basically the same, every time a positioning calculation method, small amount of calculation method 1-DEMRSSI.

\section{Positioning algorithm simulation analysis}

In order to analyze the accuracy and power consumption of the 1 - DEMMRSSI positioning algorithm in this paper, the simulation analysis based on the Mablab simulation platform. Three simulation models are established: RADAR model, 1-DEMRSSI model and the text 1-DEMMRSSI model. Simulation environment is set to base station transmit power $0 \mathrm{~dB} / \mathrm{m}$, the distance between the base station is $100 \mathrm{~m}$, signal attenuation using Abdalkarim Awad data [5].

In the simulation settings, according to the reference test points separated by the number of positioning segments 5, 10, 20, 30, 40 and simulation. The mean and standard deviation of positioning error of the three positioning methods are shown in table 2. From the simulation results, with the increase of the number of positioning segments, the positioning error of the three methods is reduced correspondingly. When the positioning section number, the proposed method has more advantages, such as positioning segment number is 5 , the average positioning error of this method was $64.2 \%$ less than the RA 2DAR method, 62.7\% less than the 1 - DEMRSSI method. Linear continuous positioning is used between two adjacent reference test points, so theoretically, this method has more advantages when the test points are few. When the number of positioning segments is longer, the positioning error mean and standard deviation of this method are less than the other two methods, the positioning accuracy can be increased by $5 \%$ 10\%. It can be seen from the simulation results that when the positioning accuracy is the same, the proposed method can greatly reduce the number of reference test points. 
Table 2 Localization error of three methods

\begin{tabular}{c|c|c|c|c|c|c|c|c|c|c|c|c}
\hline \multicolumn{10}{c}{ average (basic unit $\mathrm{m}$ ) } \\
\hline $\begin{array}{c}\text { positioning } \\
\text { segments }\end{array}$ & 5 & 10 & 20 & 30 & 40 & 50 & 5 & 10 & 20 & 30 & 40 & 50 \\
\hline $\begin{array}{c}\text { RADAR } \\
\text { method(m) }\end{array}$ & 5.49 & 3.71 & 2.25 & 1.95 & 1.83 & 1.76 & 3.62 & 2.93 & 1.79 & 1.59 & 1.52 & 1.49 \\
\hline $\begin{array}{c}\text { 1-DEMRSSI(m } \\
\text { ) }\end{array}$ & 5.28 & 2.96 & 2.02 & 1.79 & 1.69 & 1.63 & 3.34 & 2.05 & 1.49 & 1.35 & 1.29 & 1.27 \\
\hline Text method(m) & 1.97 & 1.76 & 1.64 & 1.60 & 1.57 & 1.56 & 1.36 & 1.24 & 1.23 & 1.23 & 1.23 & 1.23 \\
\hline
\end{tabular}

\section{Conclusions}

A one-dimensional localization method based on the combination of theoretical and empirical models is proposed. The positioning method firstly establishes the reference test experience model, then according to the ratio of received to locate the user of the two base station signal strength determines whether the user is located in which two adjacent test points, after linear continuous positioning. Through the simulation analysis, comparing this method with the RADAR and 1 DEMRSSI method in computing workload increases slightly when the test case, a few (such as the 4 test points), can improve the positioning accuracy of about $60 \%$, the test points is large (such as 50 test points) can increase $5 \% \sim 10 \%$. Under the same positioning accuracy, the method can greatly reduce the number of reference test points and save cost.

\section{Acknowledgments}

This work was financially supported by Research on Localization Algorithm of mobile node in Internet of things of project (project No:B2016310).

\section{References}

[1] Sun Limin, Li Jianzhong,, et al. Wireless sensor network [M] Beijing: Tsinghua University press, 2005: 135 - 155

[2] Wang Fubao, long history, Ren Fengyuan. Wireless sensor network localization systems and algorithms [J. Journal of software, 2005, 16 (5): 857 - 868

[3] BAHL P, PADMANABHAN V N. RADAR: an in - building RF - based user location and tracking system [C]. Proceedings of IEEE Infocom, Tel- Aviv: Israel, 2000: 775 - 784.

[4] Sun Qingru, Yang Xinyu, Yan Chao, et al. An improved positioning algorithm based on received signal strength indication [J] Journal of Xi'an Jiao Tong University, 2008, 42 (2): - 151 (in Chinese). ()

[5] ABDALKARIM A, THORSTEN F, FALKO D. Adap tive distance estimation and localization in W SN using RSSI measures[C]. 10 th Eurom icro Conference on D igital System Design A rchitectures, Methods and Tools, Lübeck, 2007: 471 - 478. 